penetrating highlands. Hawaii may thus provide island biogeographical data similar to 'nonequilibrial' situations such as 'mammals on mountaintops' in the southwestern USA ${ }^{13}$. There islands of boreal habitat were formed on isolated mountains when Pleistocene glaciers retreated; the numbers of small mammal species on these habitat islands seem best explained by an island biogeographical model dominated by area-dependent extinction in the absence of significant immigration or in situ speciation. Furthermore, the birds most prone to extinction in Hawaii have been those predicted for area- dependent extinction in nature reserves: large, or ecologically specialized species, or species with low powers of dispersal ${ }^{14}$. As lowland tropical rainforests around the world continue to disappear and become fragmented, it is ironic that an archipelago which has provided some of the most spectacular examples of speciation may now provide quantitative data on the rates at which species are likely to go extinct in mainland nature reserves.

Peter T. Boag is at the Edward Grey Institute, Department of Zoology, University of Oxford, Oxford OX1 3PS.

\title{
Genetics
}

\section{Control of chromosome behaviour in yeast}

\section{from Virginia A. Zakian}

EUKARYOTIC chromosomes are transmitted in a highly ordered manner during both mitosis and meiosis. For example, during each mitotic division a yeast chromosome is lost from only about 1 in $10^{5}$ cells $^{1}$. Three structures are known to be required for efficient maintenance and inheritance of eukaryotic chromosomes: (1) origins of DNA replication which allow duplication of chromosomes; (2) centromeres, which serve as sites of attachment for spindle microtubules and are required for segregation of chromosomes; and (3) telomeres, the physical ends of chromosomes which are in some way essential for the integrity of linear chromosomes. Until recently, these three structures were studied primarily by indirect procedures. Now, however, the ability to transform yeast with exogenous DNAs allows the functional cloning of sequences with the phenotypes expected for origins of DNA replication, centromeres and telomeres. This ability to clone structural domains of chromosomes has made the analysis of chromosome mechanics in yeast accessible to the most up-to-date techniques of molecular biology.

Three types of sequence have been used in recent experiments. $A R S$ s (autonomously replicating sequences) are identified by their ability to promote extrachromosomal replication of plasmid DNAs ${ }^{2}, C E N s$ (centromeres) enable replicating plasmids to segregate more efficiently during both mitosis and meiosis ${ }^{3}$, and telomeres allow plasmids to be perpetuated as linear molecules ${ }^{4}$.

One approach to understanding chromosome behaviour is to construct small artificial chromosomes in vitro containing $A R S \mathrm{~s}, C E N \mathrm{~s}$ and telomeres, as well as a structural gene to allow their selection after transformation into yeast ${ }^{5}$. Although these small (10-15 kilobases[kb]) linear $C E N$ plasmids can be maintained during both mitosis and meiosis, their mitotic stability is markedly less than that of a corresponding circular $C E N$ plasmid $^{5,6}$.

Experiments reported in a recent issue of Nature indicate that the instability of these artificial linear chromosomes is due, at least in part, to their small size ${ }^{6}$. Artificial linear chromosomes ranging in size from 10 to $56 \mathrm{~kb}$ were constructed by the addition of phage $\lambda$ DNA. After correction for copy number, the largest of these chromosomes, a molecule about one-third the size of the smallest yeast chromosome, displayed a mitotic stability very similar to that of small circular $C E N$ plasmids ${ }^{6}$. Thus, the instability of linear $C E N$ plasmids, an instability which seems to be a direct consequence of linearization ${ }^{5,6}$, can be remedied by increasing their size. However, even the $56-\mathrm{kb}$ artificial linear plasmids are unstable in mitosis compared with a bona fide yeast chromosome ${ }^{6}$.

An alternative approach to studying the biology of whole chromosomes is to introduce or delete specific segments of structural DNA in natural yeast chromosomes and ask what effect these changes have on chromosome behaviour.

Clarke and Carbon 7 used the procedure of fragment-mediated transformation ${ }^{8}$ to alter the centromere region of chromosome III. (This technique was also used by Murray and Szostak ${ }^{6}$ to construct their large artificial chromosomes.) Three types of alteration were introduced into the centromere region: deletion of $C E N 3$ sequences, inversion of $C E N 3$ relative to flanking sequences and substitution of CEN11 for CEN3 DNA. Each alteration also involved addition to the centromere region of both the structural gene $U R A 3$ (thereby providing a selectable marker for the altered chromosome) and a small portion of pBR322 DNA.

Clarke and Carbon found that deletion of a 627-base pair (bp) CEN3 fragment produces an extremely unstable chromosome, which can be maintained only in diploid cells growing under selection for expression of $U R A 3$. In contrast, inversion of $C E N 3$ or substitution of $C E N 11$ for $C E N 3$ has no measurable effect on the mitotic behaviour of chromosome III (determined from rates of chromosome loss, gene conversion and recombination) and very little, if any, effect on chromosome behaviour in meiosis.

These results provide molecular evidence which corroborates conclusions formerly based on cytological experiments (for example, refs 9,10 ); that is, that neither inversion nor replacement of a centromere alters its pairing specificity. Thus, although a centromere is required for efficient segregation, it does not function in homologue recognition. Moreover, since the terminal regions of most or all yeast chromosomes share a similar sequence content and organization ${ }^{11}$, it seems unlikely that telomere interactions could direct homologue pairing in meiosis. Rather, other unidentified structures must be responsible for homologue recognition. Since the stability of chromosomes with altered centromeres is indistinguishable from that of a wild-type yeast chromosome, all the structures responsible for duplication, pairing and segregation of yeast chromosomes must be intact and functioning on these molecules ${ }^{7}$. In contrast, the best artificial chromosomes are still $10^{2}-10^{3}$ times less stable than natural yeast chromosomes. The instability of these artificial chromosomes may be caused by any of a variety of factors, for example, the presence of foreign DNA, less than optimal size, or improper spacing of structural domains.

It is also possible, however, that as yet unidentified structures exist that influence the fidelity of DNA replication, chromosome pairing, or chromosome segregation. With the advent of transformation systems which make use of visual assays for plasmid stability ${ }^{12,13}$, this hypothesis can be tested readily by systematically scanning the yeast genome for fragments of DNA which influence the segregation of artificial chromosomes.

Virginia A. Zakian is at the Fred Hutchinson Cancer Research Center, 1124 Columbia Street Seattle, Washington 98104.

\footnotetext{
Hartwell, L.H., Dutcher, S.K., Wood, J.S. \& Garvik, B. Rec. Adv. Yeast molec. Biol. 1, 28 (1982).

2. Struhl, K., Stinchcomb, D.T., Scherer, S. \& Davis, R.W. Proc. natn. Acad. Sci. U.S. A. 76, 1035 (1979).

Clarke, L. \& Carbon, J. Nature 287, 504 (1980).

4. Szostak, J.W. \& Blackburn, E.H. Cell 29, 245 (1982)

5. Dani, G.M. \& Zakian, V.A. Proc. natn. Acad. Sci. U.S.A. 80, 3406 (1983).

. Murray, A. W \& Szostak, J W Nature 305, 189 (1983).

7. Clarke, L. \& Carbon, J. Nature 305, 23 (1983).

8. Rothstein, R.J. Meth. Enzym. 101 (in the press)

9. Sturtevant, A.H. \& Beadle, G.W. Genetics 21, 554 (1936).

10. Sandler, L. Drosoph. Inf. Serv. 30, 150 (1956).

II. Chan, C.S.M. \& Tye, B-K. Cell 33, 563 (1983).

12. Hieter, P., Mann, C., Snyder, M. \& Davis, R.W (unpublished data)
}

13. Koshland, D. \& Hartwell, L. (unpublished data). 